\title{
Brief communication: Firn data compilation reveals the evolution of the firn air content on the Greenland ice sheet
}

Baptiste Vandecrux ${ }^{1,2}$, Michael MacFerrin ${ }^{3}$, Horst Machguth ${ }^{4,5}$, William T. Colgan ${ }^{1}$, Dirk van As ${ }^{1}$, Achim Heilig $^{6}$, C. Max Stevens ${ }^{7}$, Charalampos Charalampidis ${ }^{8}$, Robert S. Fausto ${ }^{1}$, Elizabeth M. Morris $^{9}$, Ellen Mosley-Thompson ${ }^{10}$, Lora Koenig ${ }^{11}$, Lynn N. Montgomery ${ }^{11}$, Clément Miège ${ }^{12}$, Sebastian B. Simonsen ${ }^{13}$, Thomas Ingeman-Nielsen ${ }^{2}$, Jason E. Box ${ }^{1}$

${ }^{1}$ Geological Survey of Denmark and Greenland, Copenhagen, Denmark.

${ }^{2}$ Department of Civil Engineering, Technical University of Denmark, Lyngby, Denmark.

${ }^{3}$ Cooperative Institute for Research in Environmental Sciences, University of Colorado, Boulder, CO USA

${ }^{4}$ Department of Geosciences, University of Fribourg, Fribourg, Switzerland

${ }^{5}$ Department of Geography, University of Zurich, Zurich, Switzerland

${ }^{6}$ Department of Earth and Environmental Sciences, LMU, Munich, Germany

${ }^{7}$ Department of Earth and Space Sciences, University of Washington, WA USA

${ }^{8}$ Bavarian Academy of Sciences and Humanities, Munich, Germany

${ }^{9}$ Scott Polar Research Institute, Cambridge University, United Kingdom

${ }_{10}$ Byrd Polar and Climate Research Center and Department of Geography, Ohio State University, Columbus, OH USA.

${ }^{11}$ National Snow and Ice Data Center, University of Colorado, Boulder, NV, United States

${ }^{12}$ Department of Geography, Rutgers University, Piscataway, NJ, United States

${ }^{13}$ National Space Institute, Technical University of Denmark, Kgs. Lyngby, Denmark

Correspondence to: B. Vandecrux (bava@byg.dtu .dk)
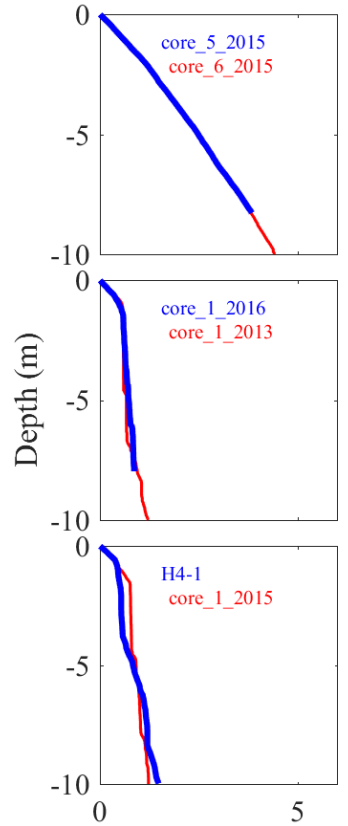
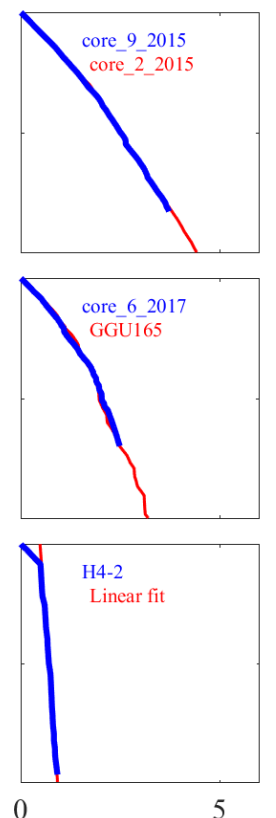
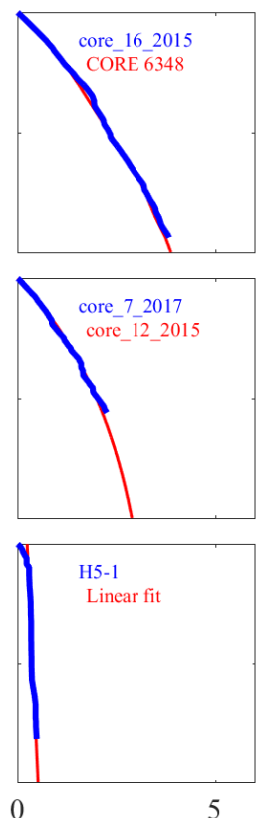
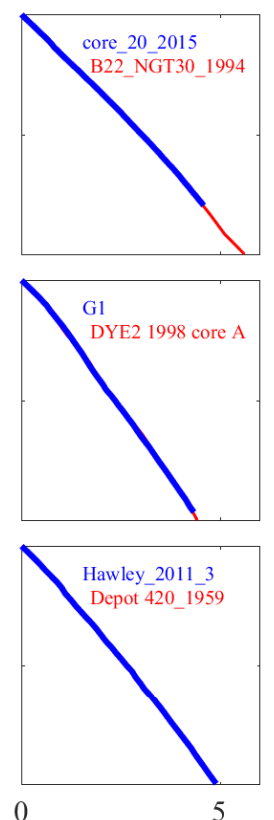
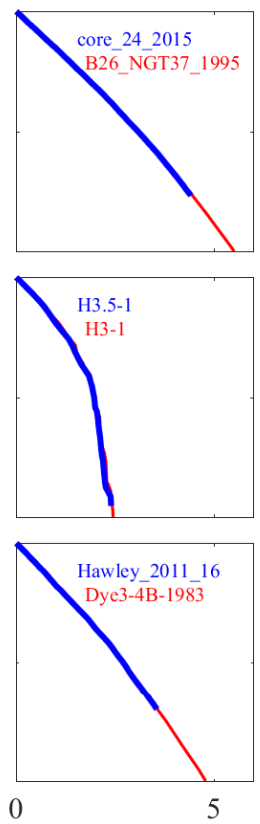

Cumulated firn air content $\left(\mathrm{m}^{3} \mathrm{~m}^{-2}\right)$

Figure S1. Extrapolation of FAC to $10 \mathrm{~m}$ using the lower section of the existing $\mathbf{1 0} \mathbf{m}$-long FAC profile that has the lowest Root Mean Squared Difference with the shallow FAC profile. 

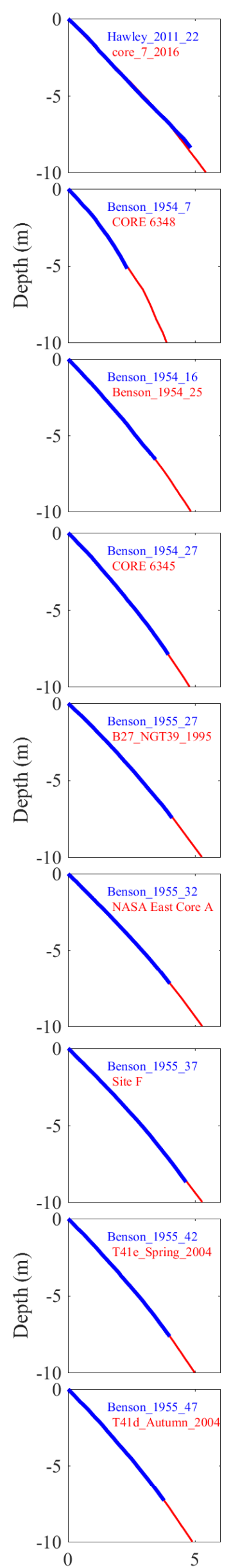
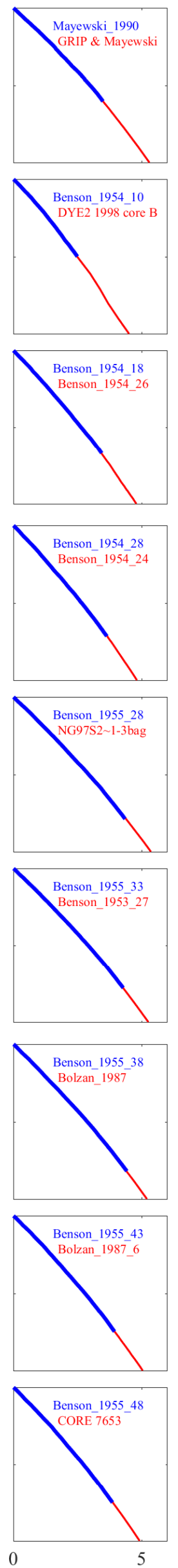
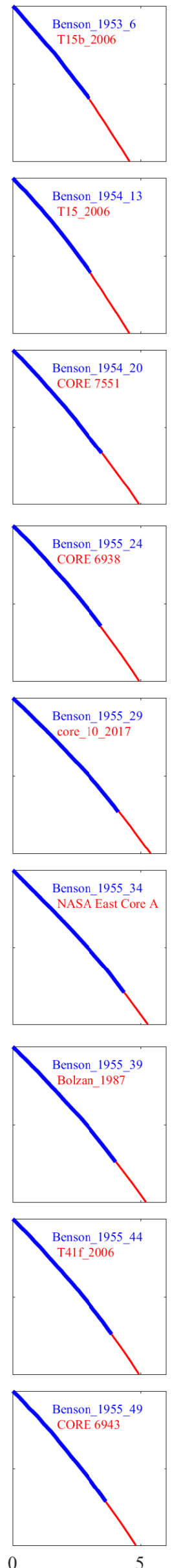
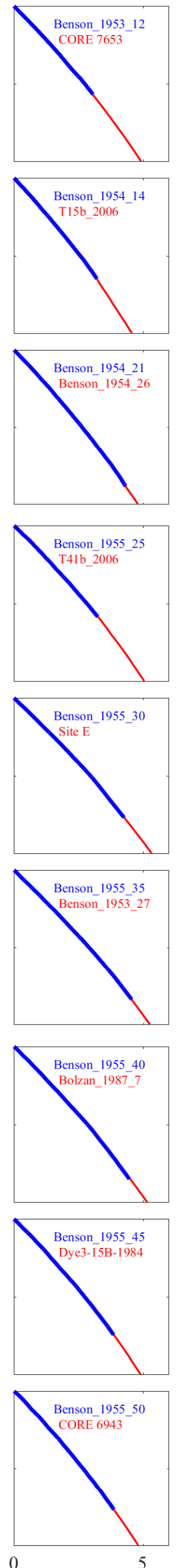

Cumulated firn air content $\left(\mathrm{m}^{3} \mathrm{~m}^{-2}\right)$
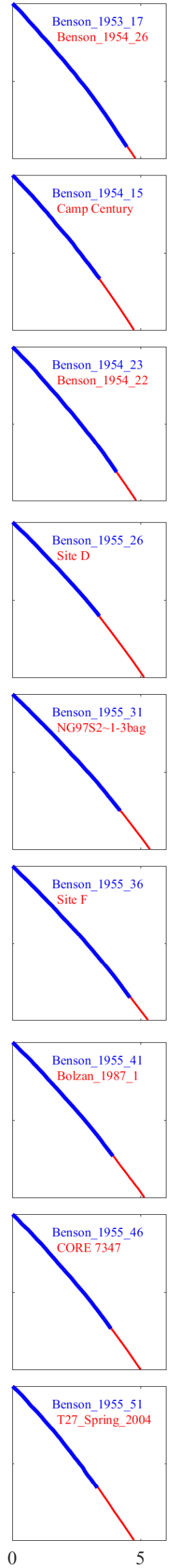

Figure S1. Extrapolation of FAC to $10 \mathrm{~m}$ using the lower section of the existing $10 \mathrm{~m}$-long FAC profile that has the lowest Root Mean Squared Difference with the shallow FAC profile. 

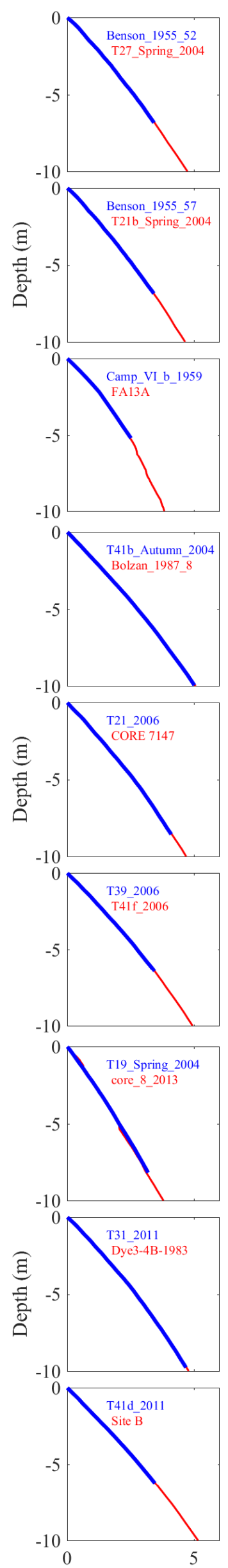
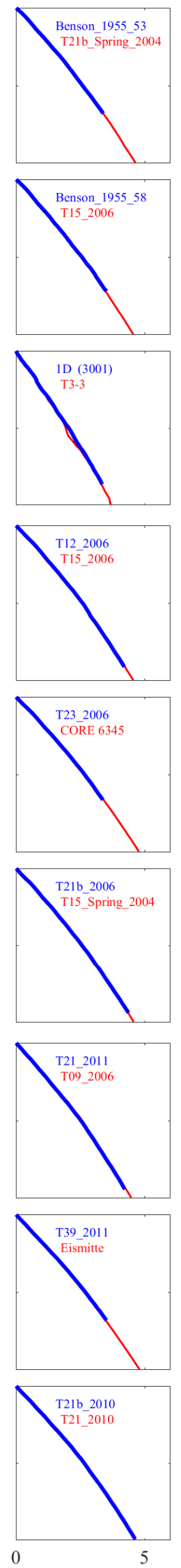
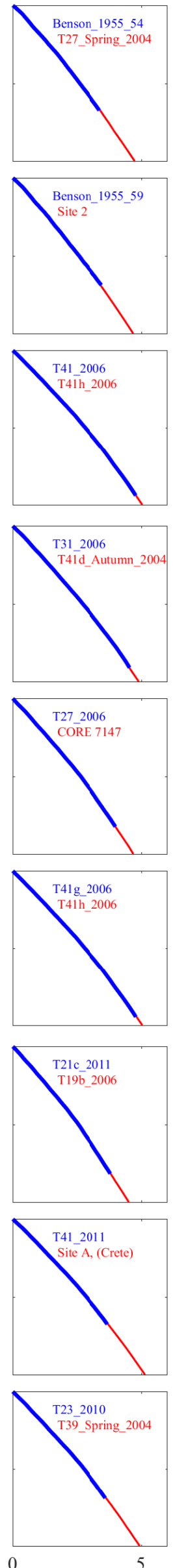
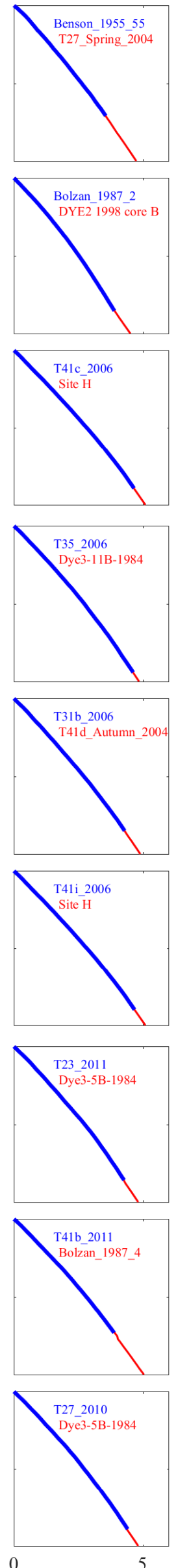

Cumulated firn air content $\left(\mathrm{m}^{3} \mathrm{~m}^{-2}\right)$
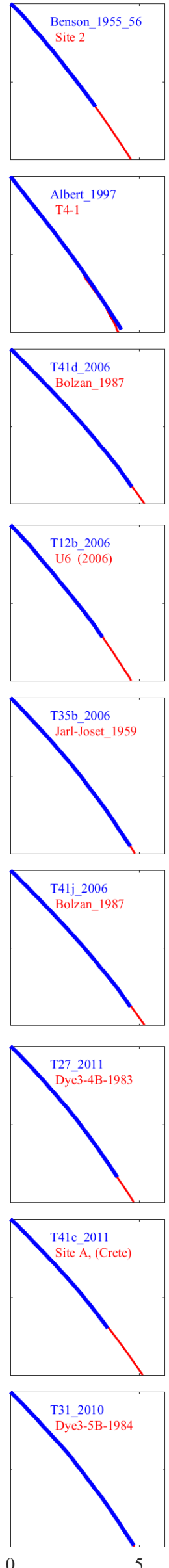

Figure S1. Extrapolation of FAC to $10 \mathrm{~m}$ using the lower section of the existing $\mathbf{1 0} \mathbf{~ m}$-long FAC profile that has the lowest Root Mean Squared Difference with the shallow FAC profile. 

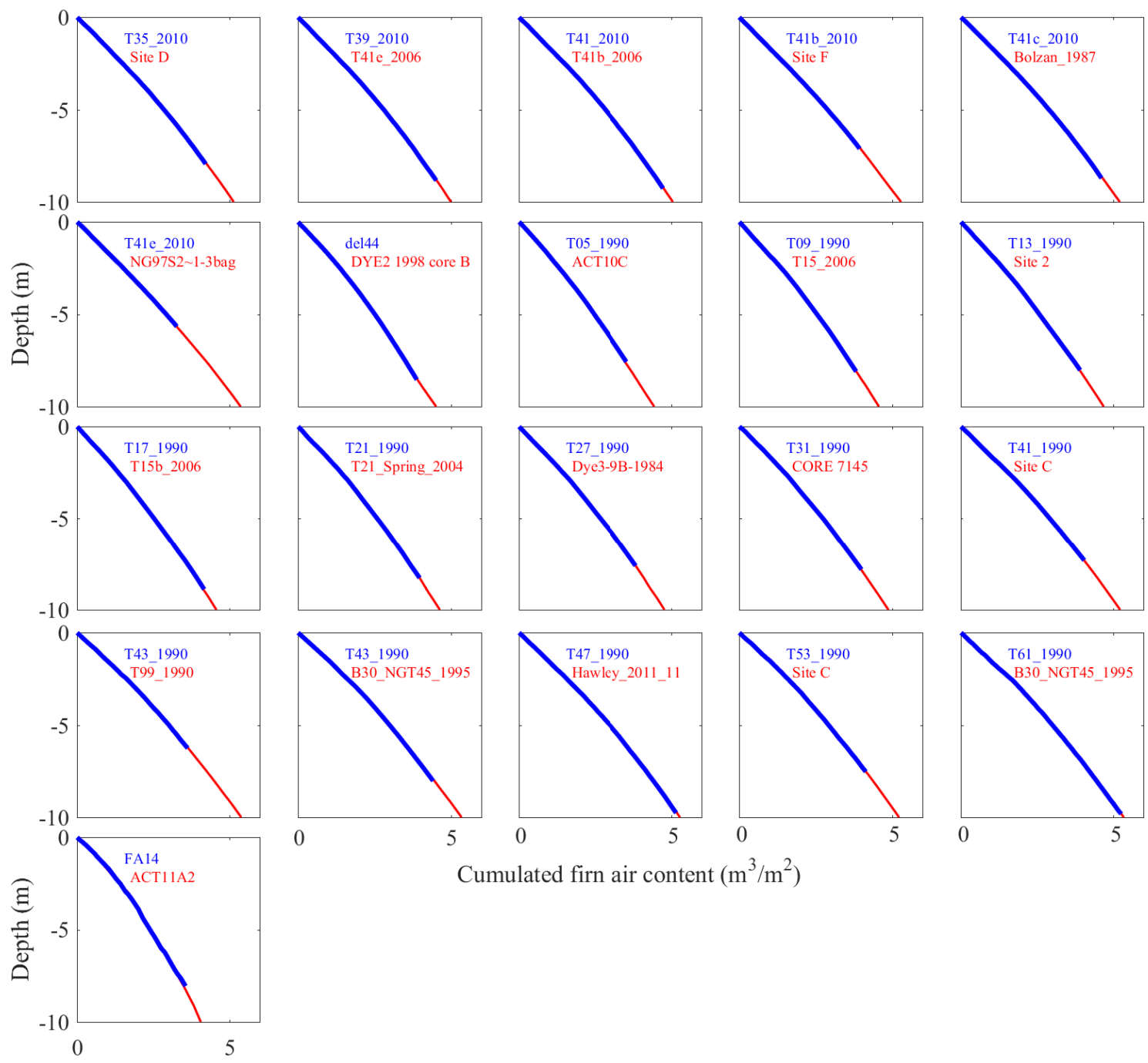

Cumulated firn air content $\left(\mathrm{m}^{3} / \mathrm{m}^{2}\right)$

Figure S1. Extrapolation of FAC to $10 \mathrm{~m}$ using the lower section of the existing $\mathbf{1 0} \mathbf{~ m}$-long FAC profile that has the lowest Root Mean Squared Difference with the shallow FAC profile.
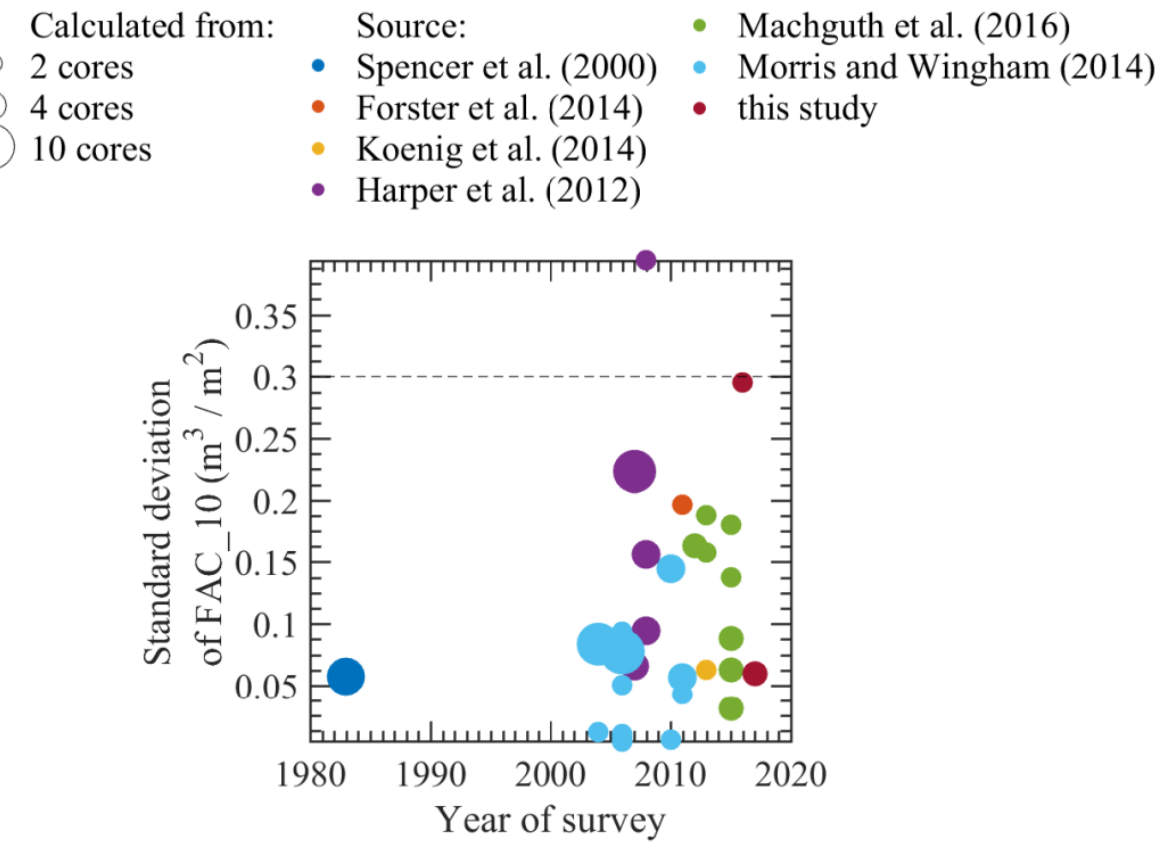

Figure S2. Standard deviation of FAC measurements located within $1 \mathrm{~km}$. Dashed line indicate $0.3 \mathrm{~m}^{3} \mathrm{~m}^{-2}$, value used to describe the uncertainty applying on any $\mathrm{FAC}_{10}$ measurement. 
a) Dry Snow Area

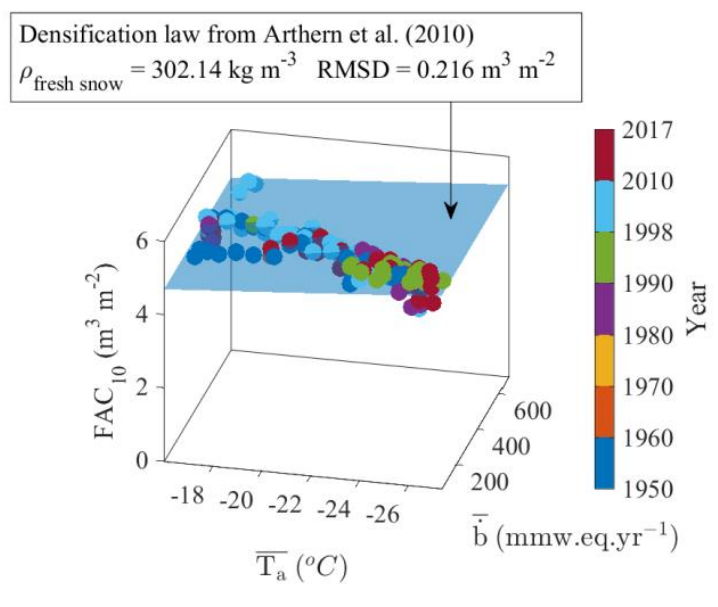

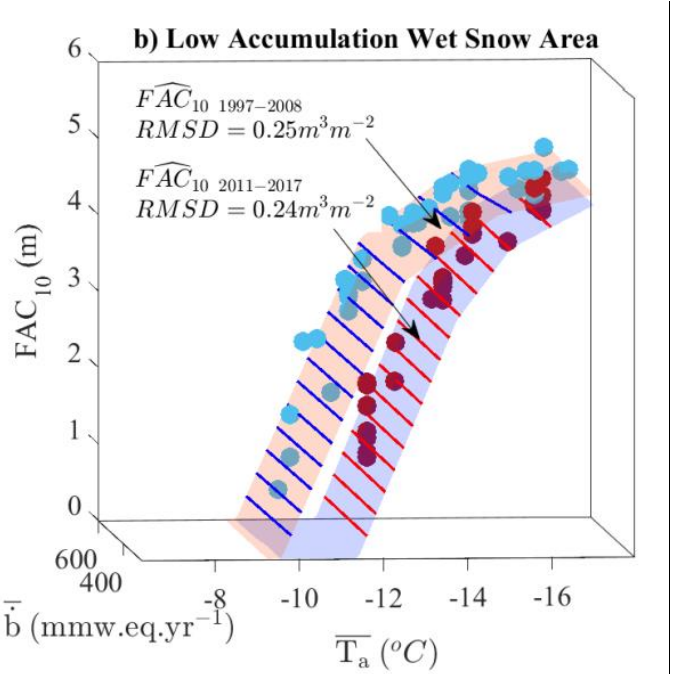

$\overline{\mathrm{T}_{\mathrm{a}}}\left({ }^{o} \mathrm{C}\right)$

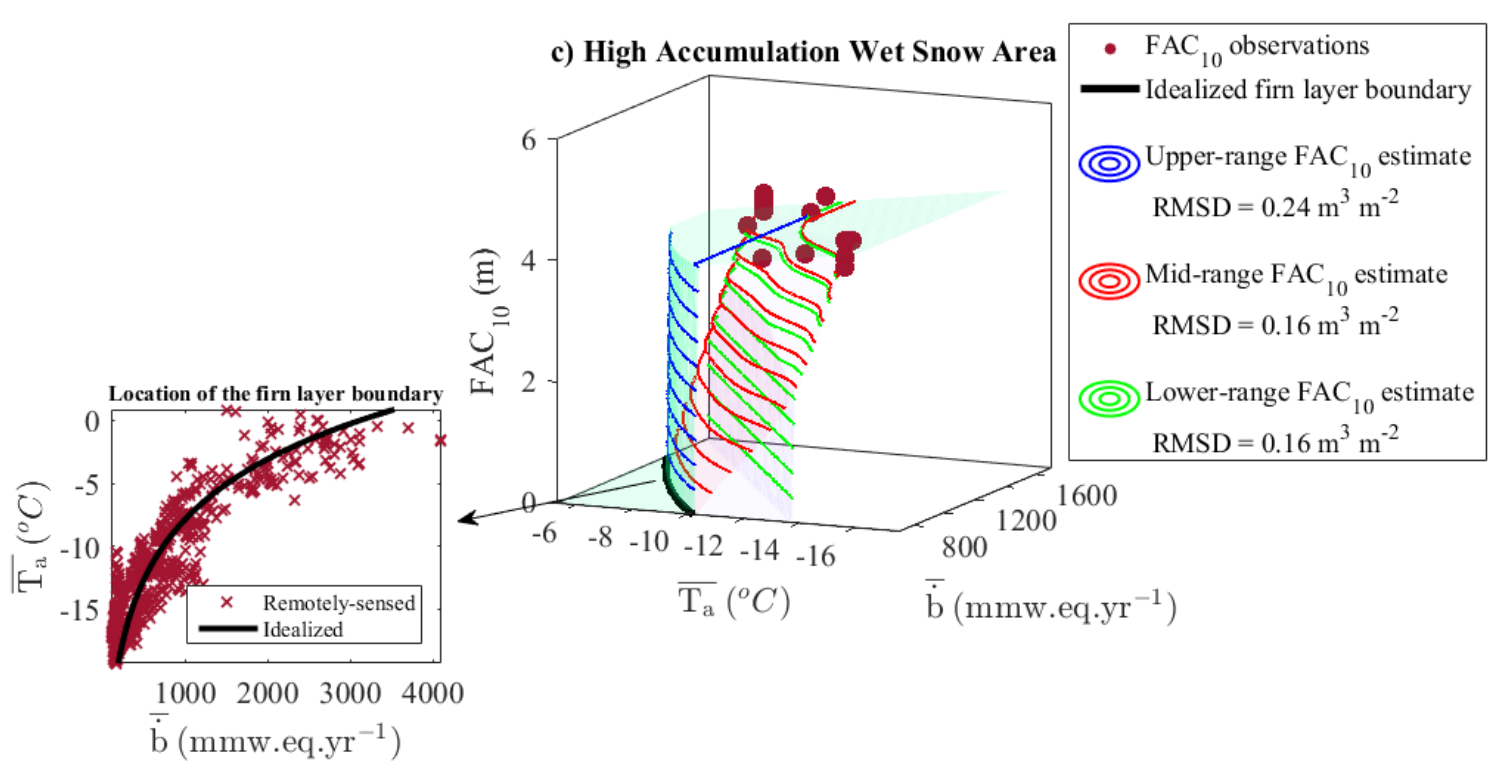

Figure S3. Empirical functions of $\overline{\dot{b}}$ and $\overline{T_{a}}$, fitted to the $\mathrm{FAC}_{10}$ observations and used to predict $\mathrm{FAC}_{10}$ over the entire frin area. a) Over the DSA, the firn densification function from Arthern et al. (2010) (blue plane) was used with a fresh snow density of $302.15 \mathrm{~kg} \mathrm{~m}^{-3}$. b) In the LAWSA two functions linearly increasing with $\bar{b}$ and piecewise linearly decreasing with $\overline{T_{a}}$ (two slope breaks at $1 / 3$ and $2 / 3$ of the $\overline{T_{a}}$ range covered by the data) were fitted separately to the $F A C_{10}$ observations for 1997-2008 and 2011-2017 periods. c) In the HAWSA we use the linear dependence of FAC $\mathrm{C}_{10}$ on $\overline{T_{a}}$ (Section 2.5.3.) as maximum value. The upper-range $\mathrm{FAC}_{10}$ estimate (blue contour lines) follows that linear function of $\frac{\bar{T}}{T_{a}}$ until it reaches the idealized boundary of the firn area (black line, exponential fit to the remotely-sensed location of the firn line in the $\left(\overline{\dot{b}}, \overline{T_{a}}\right)$ space, inset). The mid-range estimate links the measurements and the idealized boundary of the firn area using a smoothed bilinear function. The lower-range estimate follows the mid-range estimate in the lowest temperatures but is set to zero for any location where temperature exceeds the $\mathrm{FAC}_{10}$ measurements by $1^{\circ} \mathrm{C}$. 


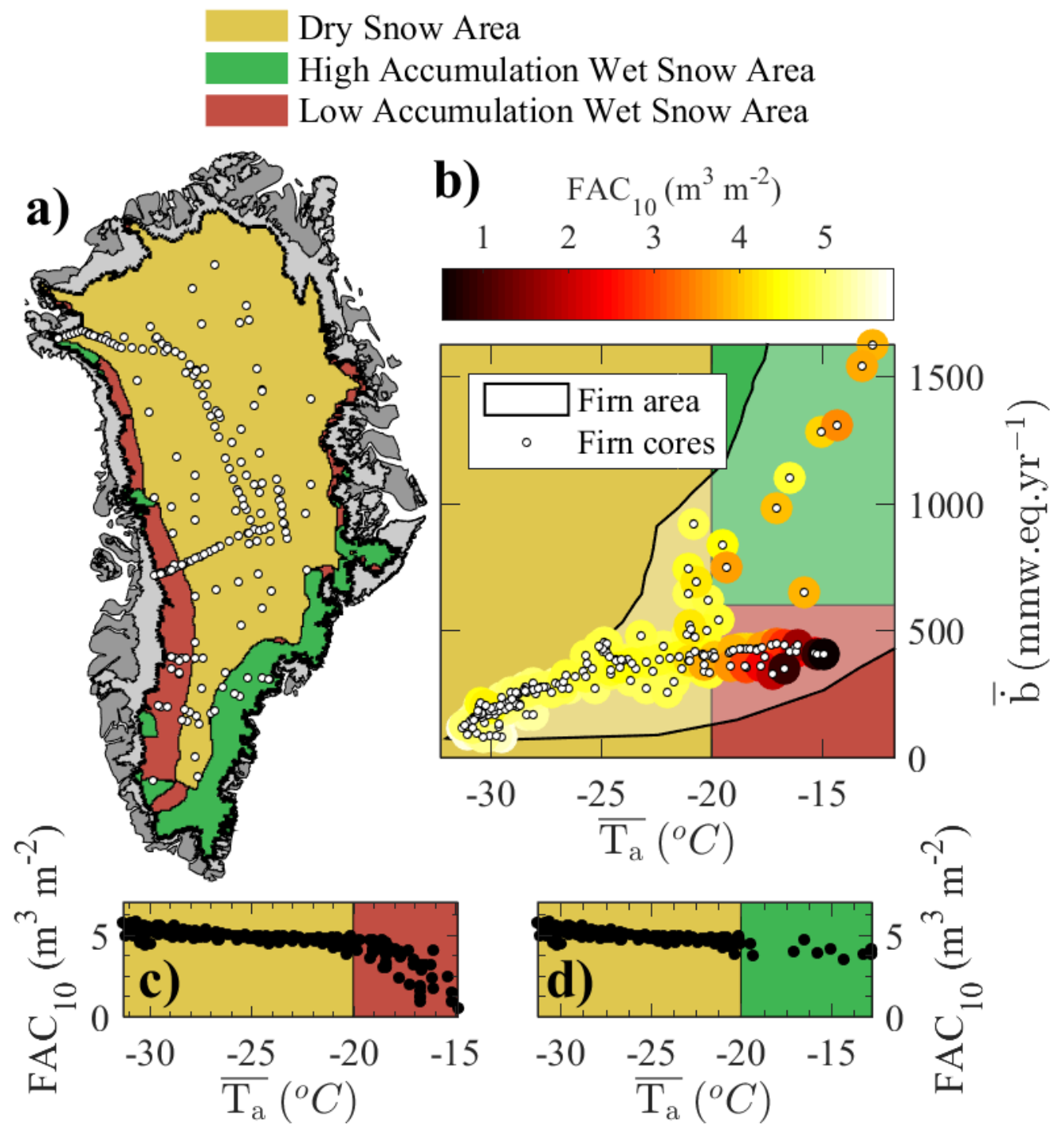

Figure S4. Repetition of the procedure with $\bar{b}$ and $\overline{T_{a}}$ taken as 1979-2014 average snowfall and surface temperature from MAR regional climate model (Fettweis et al. 2017). Threshold temperature between the dry snow area and the wet snow areas is $-\mathbf{2 0}^{\circ} \mathrm{C}$ given the different definition of $\overline{T_{a}}$. a) Geographical distribution of the $\mathrm{FAC}_{10}$ dataset. B) Distribution of the dataset in the accumulation-temperature space $\left(\bar{b}\right.$ and $\left.\overline{T_{a}}\right)$. FAC 10 value is indicated by a coloured disk around each point. c) Temperature dependency of FAC $_{10}$ in the DSA and LAWSA d) Temperature dependency of FAC 10 in the DSA and HAWSA. 
a) Dry Snow Area

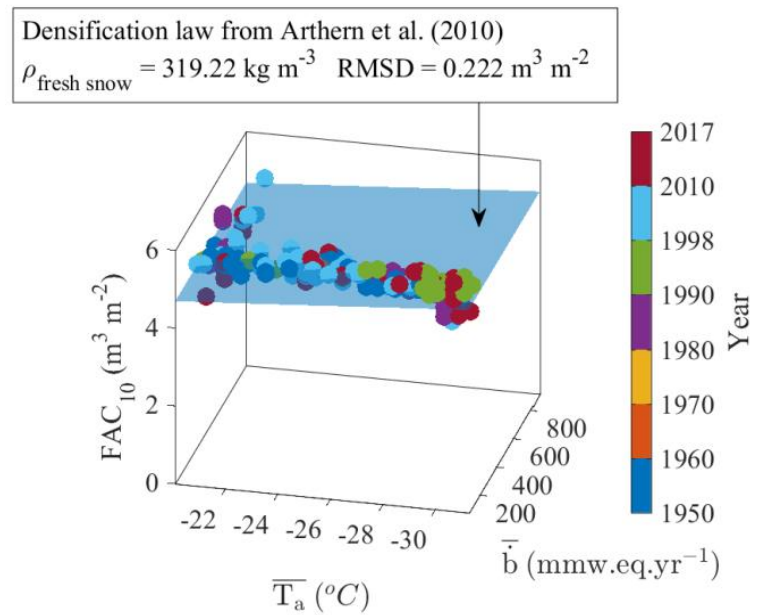

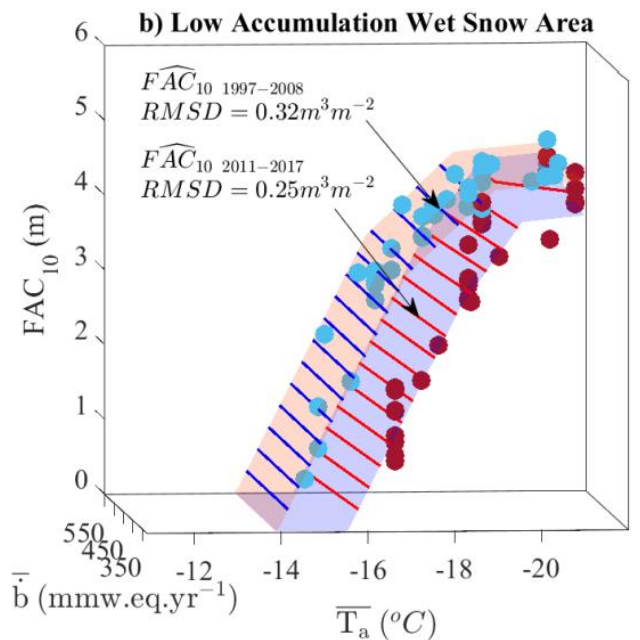

$\overline{\mathrm{T}_{\mathrm{a}}}\left({ }^{\circ} \mathrm{C}\right)$

c) High Accumulation Wet Snow Area

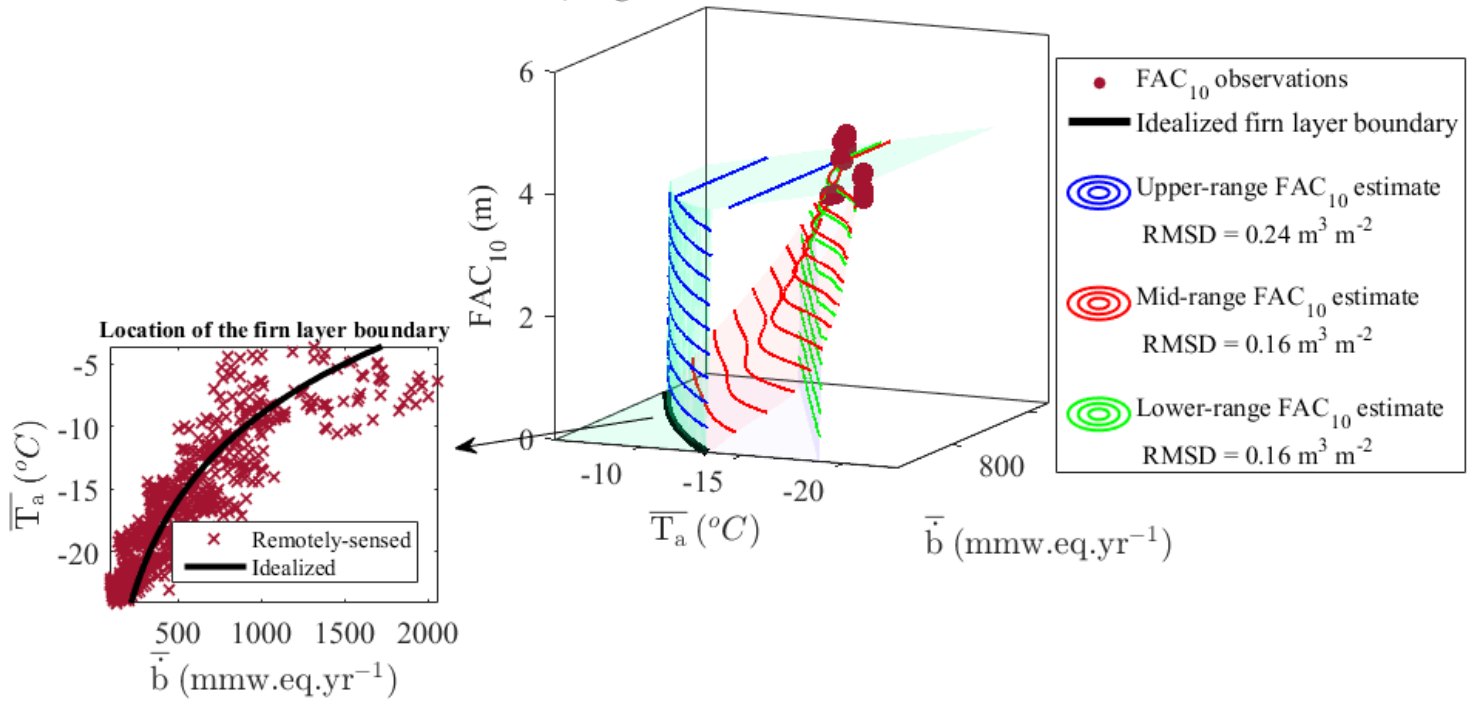

Figure S5. Construction of the empirical functions used for the mapping of $\mathrm{FAC}_{10}$, similar to Figure $\mathrm{S3}$ but using $\overline{\dot{b}}$ and $\overline{T_{a}}$ from MAR (Fettweis et al. 2017).
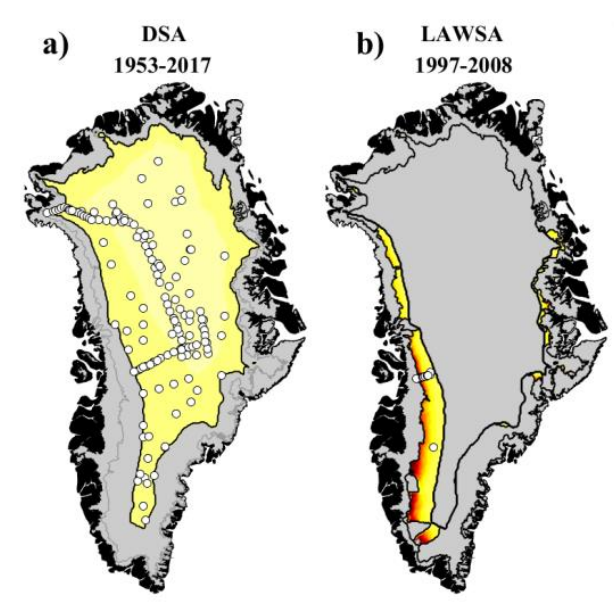

LAWSA 2011-2017
$\&$ HAWSA 2010-2017

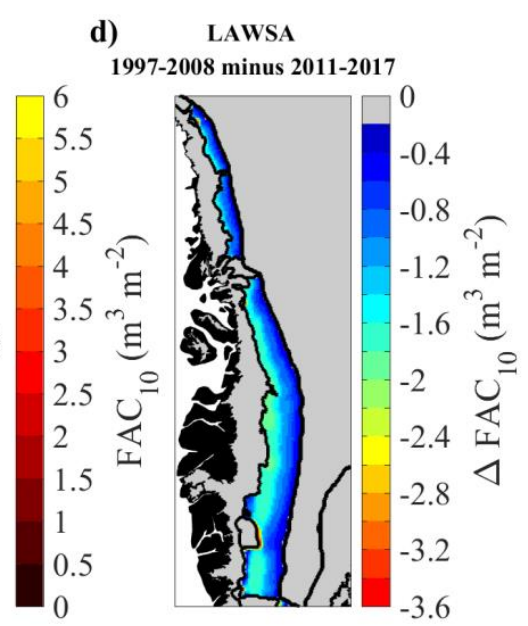

Figure S6. FAC ${ }_{10}$ maps using the $\bar{b}$ and $\overline{T_{a}}$ maps from MAR (Fettweis et al. 2017). 


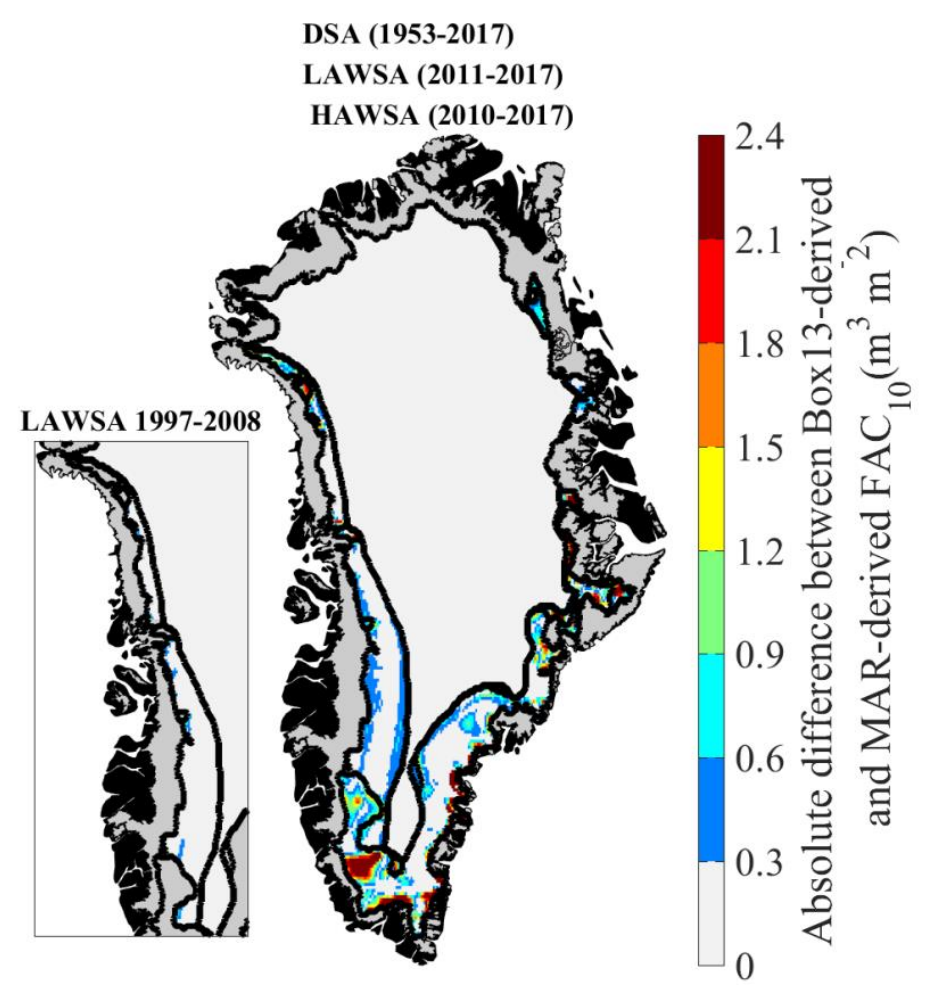

Figure S7. Difference between the $\mathrm{FAC}_{10}$ maps obtained using $\overline{\dot{b}}$ and $\bar{T}_{a}$ from Box (2013) and Box et al. (2013) and the maps obtained using $\overline{\dot{b}}$ and $\overline{T_{a}}$ from MAR (Fettweis et al. 2017). 


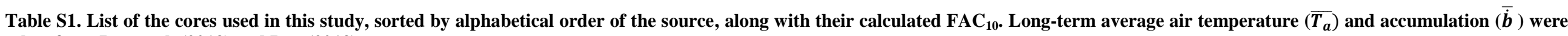
taken from Box et al. (2013) and Box (2013).

\begin{tabular}{|c|c|c|c|c|c|c|c|c|}
\hline Year & Name & $\begin{array}{l}\text { Latitude } \\
\qquad\left({ }^{\circ} \mathrm{N}\right)\end{array}$ & $\begin{array}{l}\text { Longitude } \\
\qquad\left({ }^{\circ} \mathrm{E}\right)\end{array}$ & $\begin{array}{l}\overline{T_{a}} \\
\left({ }^{\circ} \mathrm{C}\right)\end{array}$ & $\begin{array}{c}\overline{\dot{b}}(\mathrm{~mm} \\
\left.\text { w.eq. } \mathrm{yr}^{-1}\right)\end{array}$ & $\begin{array}{l}\text { Length of } \\
\text { core }(\mathrm{m})\end{array}$ & $\begin{array}{l}\mathrm{FAC}_{10} \\
\left(\mathrm{~m}^{3} \mathrm{~m}^{-2}\right)\end{array}$ & Source \\
\hline 1997 & Albert_1997 & 72.58 & -38.50 & -30.2 & 204 & 9.8 & 4.4 & $\begin{array}{l}\text { Albert, M., and Shultz, E.: Snow and firn properties and air- } \\
\text { snow transport processes at Summit, Greenland, Atmos. } \\
\text { Environ., 36, 2789-2797, } 2002 .\end{array}$ \\
\hline 1987 & Site A & 70.63 & -35.82 & -28.0 & 293 & 109.0 & 5.1 & $\begin{array}{l}\text { Alley, R.: Transformations in Polar Firn, Ph.D. Thesis, } \\
\text { University of Wisconsin, Madison, WI, USA, } 1987 .\end{array}$ \\
\hline 1954 & Eismitte & 71.75 & -40.75 & -27.8 & 272 & 15.0 & 4.8 & $\begin{array}{l}\text { Bader, H.: Sorge's law of densication of snow on high polar } \\
\text { glaciers, Journal of Glaciology }, 2,15,319-411,1954 \text {. }\end{array}$ \\
\hline 2009 & Baker_2009 & 77.45 & -51.06 & -28.6 & 210 & 79.8 & 5.2 & $\begin{array}{l}\text { Baker, I.: Density and permeability measurements with depth } \\
\text { for the NEEM 2009S2 firn core, ACADIS Gateway, } \\
\text { doi:10.18739/A2Q88G, } 2012 \text {. }\end{array}$ \\
\hline 1954 & Benson_1954_12 & 76.72 & -65.39 & -19.7 & 543 & 14.4 & 4.7 & \multirow{17}{*}{$\begin{array}{l}\text { Benson, C. S.: Stratigraphic Studies in the Snow and Firn of } \\
\text { the Greenland Ice Sheet, U.S. Army Snow, Ice and Permafrost } \\
\text { Research Establishment, } 1962 \text {. }\end{array}$} \\
\hline 1954 & Benson_1954_14 & 76.89 & -64.40 & -21.0 & 379 & 6.5 & 4.6 & \\
\hline 1954 & Benson_1954_15 & 76.96 & -63.90 & -21.4 & 347 & 6.7 & 4.8 & \\
\hline 1954 & Benson_1954_16 & 77.05 & -63.39 & -21.7 & 296 & 6.6 & 4.9 & \\
\hline 1954 & Benson_1954_19 & 77.24 & -62.33 & -22.9 & 270 & 16.8 & 4.9 & \\
\hline 1954 & Benson_1954_20 & 77.24 & -61.67 & -23.7 & 306 & 6.7 & 4.9 & \\
\hline 1954 & Benson_1954_21 & 77.22 & -61.02 & -24.3 & 382 & 8.9 & 4.8 & \\
\hline 1954 & Benson_1954_22 & 77.18 & -60.39 & -24.9 & 417 & 15.2 & 4.8 & \\
\hline 1954 & Benson_1954_23 & 77.18 & -59.74 & -24.9 & 453 & 8.2 & 4.8 & \\
\hline 1954 & Benson_1954_24 & 77.15 & -59.09 & -25.1 & 436 & 10.2 & 4.8 & \\
\hline 1954 & Benson_1954_25 & 77.13 & -58.45 & -25.0 & 435 & 10.3 & 4.8 & \\
\hline 1954 & Benson_1954_26 & 77.09 & -57.82 & -24.7 & 446 & 14.2 & 4.8 & \\
\hline 1954 & Benson_1954_27 & 77.06 & -57.20 & -24.9 & 429 & 7.9 & 4.8 & \\
\hline 1954 & Benson_1954_28 & 77.03 & -56.56 & -24.8 & 411 & 7.2 & 4.8 & \\
\hline 1953 & Benson_1953_6 & 76.73 & -65.42 & -20.2 & 500 & 6.0 & 4.6 & \\
\hline 1953 & Benson_1953_12 & 77.24 & -62.33 & -22.9 & 270 & 5.7 & 4.9 & \\
\hline 1953 & Benson_1953_17 & 77.15 & -59.10 & -25.1 & 436 & 9.3 & 4.8 & \\
\hline
\end{tabular}




$\begin{array}{llllllll}1953 & \text { Benson_1953_27 } & 77.30 & -49.94 & -29.0 & 206 & 10.8 & 5.3 \\ 1955 & \text { Benson_1955_24 } & 77.03 & -54.52 & -25.8 & 339 & 6.5 & 5.0 \\ 1955 & \text { Benson_1955_25 } & 77.05 & -52.92 & -26.9 & 287 & 5.9 & 5.1 \\ 1955 & \text { Benson_1955_26 } & 77.05 & -51.33 & -27.9 & 250 & 6.1 & 5.2 \\ 1955 & \text { Benson_1955_27 } & 77.07 & -49.60 & -28.9 & 217 & 7.5 & 5.2 \\ 1955 & \text { Benson_1955_28 } & 77.07 & -48.02 & -29.6 & 182 & 7.9 & 5.3 \\ 1955 & \text { Benson_1955_29 } & 76.97 & -46.98 & -30.1 & 170 & 7.3 & 5.4 \\ 1955 & \text { Benson_1955_30 } & 76.64 & -45.70 & -30.4 & 166 & 7.7 & 5.3 \\ 1955 & \text { Benson_1955_31 } & 76.32 & -45.10 & -30.5 & 171 & 7.5 & 5.4 \\ 1955 & \text { Benson_1955_32 } & 75.99 & -44.58 & -30.5 & 175 & 7.2 & 5.3 \\ 1955 & \text { Benson_1955_33 } & 75.64 & -43.95 & -30.4 & 175 & 7.8 & 5.3 \\ 1955 & \text { Benson_1955_34 } & 75.30 & -43.42 & -30.4 & 177 & 7.9 & 5.3 \\ 1955 & \text { Benson_1955_35 } & 74.94 & -42.97 & -30.3 & 179 & 8.4 & 5.3 \\ 1955 & \text { Benson_1955_36 } & 74.59 & -42.55 & -30.0 & 182 & 8.6 & 5.3 \\ 1955 & \text { Benson_1955_37 } & 74.22 & -42.17 & -29.9 & 185 & 8.7 & 5.3 \\ 1955 & \text { Benson_1955_38 } & 74.87 & -41.80 & -30.8 & 149 & 8.2 & 5.2 \\ 1955 & \text { Benson_1955_39 } & 73.52 & -41.42 & -29.3 & 188 & 7.4 & 5.2 \\ 1955 & \text { Benson_1955_40 } & 73.17 & -41.10 & -29.2 & 199 & 8.5 & 5.1 \\ 1955 & \text { Benson_1955_41 } & 72.82 & -40.75 & -29.1 & 219 & 7.3 & 5.1 \\ 1955 & \text { Benson_1955_42 } & 72.47 & -40.33 & -29.0 & 232 & 7.7 & 5.0 \\ 1955 & \text { Benson_1955_43 } & 72.12 & -39.93 & -28.9 & 241 & 7.5 & 5.1 \\ 1955 & \text { Benson_1955_44 } & 71.77 & -39.60 & -28.8 & 249 & 7.4 & 5.0 \\ 1955 & \text { Benson_1955_45 } & 71.43 & -39.33 & -28.3 & 258 & 7.5 & 4.9 \\ 1955 & \text { Benson_1955_46 } & 71.08 & -38.97 & -28.0 & 266 & 7.4 & 5.0 \\ 1955 & \text { Benson_1955_47 } & 71.00 & -39.67 & -27.5 & 277 & 7.3 & 4.9 \\ 1955 & \text { Benson_1955_48 } & 70.91 & -40.63 & -26.7 & 296 & 7.5 & 4.9 \\ 1955 & \text { Benson_1955_49 } & 70.78 & -41.63 & -25.7 & 318 & 7.2 & 4.8 \\ 1955 & \text { Benson_1955_50 } & 70.63 & -42.62 & -24.6 & 344 & 7.7 & 4.8 \\ 1955 & \text { Benson_1955_51 } & 70.46 & -43.58 & -23.6 & 369 & 6.6 & 4.7 \\ 1955 & \text { Benson_1955_52 } & 70.30 & -44.55 & -22.4 & 388 & 6.8 & 4.7 \\ 1955 & \text { Benson_1955_53 } & 70.25 & -44.97 & -21.9 & 391 & 6.8 & 4.7\end{array}$




\begin{tabular}{|c|c|c|c|c|c|c|c|c|}
\hline 1955 & Benson_1955_54 & 70.18 & -45.37 & -21.5 & 398 & 6.8 & 4.7 & \\
\hline 1955 & Benson_1955_55 & 70.11 & -45.73 & -21.0 & 402 & 7.1 & 4.8 & \\
\hline 1955 & Benson_1955_56 & 70.04 & -46.13 & -20.4 & 401 & 6.6 & 4.7 & \\
\hline 1955 & Benson_1955_57 & 69.98 & -46.50 & -19.9 & 411 & 6.9 & 4.7 & \\
\hline 1955 & Benson_1955_58 & 69.92 & -46.93 & -19.1 & 418 & 7.2 & 4.6 & \\
\hline 1955 & Benson_1955_59 & 69.87 & -47.30 & -18.6 & 428 & 6.9 & 4.7 & \\
\hline 1987 & Bolzan_1987 & 77.98 & -37.70 & -30.3 & 79 & 16.6 & 5.2 & \multirow{9}{*}{$\begin{array}{l}\text { Bolzan, J. F., and Strobel, M.: Oxygen isotope data from } \\
\text { snowpit at GISP2 Site 15., PANGAEA, } \\
\text { doi:10.1594/PANGAEA.55511, } 1999 .\end{array}$} \\
\hline 1987 & Bolzan_1987_1 & 72.21 & -35.67 & -29.8 & 199 & 14.8 & 5.2 & \\
\hline 1987 & Bolzan_1987_2 & 72.29 & -37.92 & -30.4 & 215 & 8.5 & 4.5 & \\
\hline 1987 & Bolzan_1987_3 & 71.60 & -38.14 & -29.5 & 237 & 16.9 & 5.1 & \\
\hline 1987 & Bolzan_1987_4 & 71.92 & -35.96 & -29.6 & 207 & 11.0 & 5.0 & \\
\hline 1987 & Bolzan_1987_5 & 72.64 & -35.94 & -30.6 & 186 & 14.2 & 5.1 & \\
\hline 1987 & Bolzan_1987_6 & 72.35 & -40.21 & -29.0 & 235 & 16.5 & 5.1 & \\
\hline 1987 & Bolzan_1987_7 & 72.89 & -39.16 & -30.0 & 193 & 16.2 & 5.2 & \\
\hline 1987 & Bolzan_1987_8 & 71.93 & -39.84 & -28.7 & 249 & 16.5 & 5.1 & \\
\hline 2011 & B262011 & 77.25 & -49.21 & -29.4 & 193 & 29.7 & 5.4 & \multirow{8}{*}{$\begin{array}{l}\text { Buchardt, S. L., Clausen, H. B., Vinther, B. M., and Dahl- } \\
\text { Jensen, D.: Investigating the past and recent delta 18O- } \\
\text { accumulation relationship seen in Greenland ice cores, Clim. } \\
\text { Past, 8, 6, 2053-2059, } 2012 .\end{array}$} \\
\hline 2011 & Camp Century 2010 & 77.85 & -52.02 & -28.3 & 168 & 40.7 & 5.3 & \\
\hline 2010 & CC_2010_ & 77.18 & -60.50 & -24.8 & 431 & 30.3 & 5.0 & \\
\hline 2007 & TraverseSite1 & 76.45 & -44.77 & -30.7 & 157 & 70.7 & 5.4 & \\
\hline 2001 & NGRIP2001S4 & 75.10 & -42.30 & -30.7 & 157 & 100.1 & 5.5 & \\
\hline 2001 & NGRIP2001S5 & 75.10 & -42.30 & -30.7 & 157 & 100.1 & 5.5 & \\
\hline 1997 & NG97S2 1-3bag & 75.10 & -42.30 & -30.7 & 157 & 141.5 & 5.4 & \\
\hline 1987 & del44 & 72.29 & -35.92 & -29.9 & 197 & 8.5 & 4.5 & \\
\hline 1988 & Site A, (Crete) & 71.12 & -37.32 & -29.1 & 256 & 128.6 & 5.2 & \multirow{8}{*}{$\begin{array}{l}\text { Clausen, H., Gundestrup, N. S., Johnsen, S. J., Binchadler, R., } \\
\text { and Zwally, J.: Glaciological investigations in the Crete area, } \\
\text { Central Greenland: a search for a new deep-drilling Site, Ann. } \\
\text { Glaciol., 10,10-15, } 1988 .\end{array}$} \\
\hline 1988 & Site B & 70.65 & -37.48 & -28.4 & 279 & 105.6 & 5.2 & \\
\hline 1988 & Site C & 70.68 & -38.79 & -27.7 & 279 & 24.8 & 5.2 & \\
\hline 1988 & Site D & 70.64 & -39.62 & -27.0 & 291 & 100.1 & 5.1 & \\
\hline 1988 & Site E & 71.76 & -35.85 & -29.4 & 212 & 77.8 & 5.3 & \\
\hline 1988 & Site F & 71.49 & -35.90 & -29.2 & 221 & 25.7 & 5.3 & \\
\hline 1988 & Site $G$ & 71.15 & -35.84 & -28.8 & 239 & 70.8 & 5.2 & \\
\hline 1988 & Site H & 70.87 & -35.84 & -28.5 & 267 & 26.2 & 5.1 & \\
\hline
\end{tabular}




\begin{tabular}{|c|c|c|c|c|c|c|c|c|}
\hline 2017 & Camp Century 2017 & 77.17 & -61.13 & -24.3 & 382 & 62.3 & 5.0 & $\begin{array}{l}\text { Colgan, W., Pedersen, A., Binder, D., Machguth, H., } \\
\text { Abermann, J., and Jayred, M.: Initial field activities of the } \\
\text { Camp Century Climate Monitoring Programme in Greenland. } \\
\text { Geological Survey of Denmark and Greenland Bulletin, Geol. } \\
\text { Surv. Denmark Greenland Bull., 41, 75-78, } 2018 .\end{array}$ \\
\hline 1990 & T05_1990 & 69.85 & -47.25 & -18.7 & 425 & 7.6 & 4.5 & \multirow{14}{*}{$\begin{array}{l}\text { Fischer, H., Wagenbach, D., Laternser, M., and Haeberli, W.: } \\
\text { Glacio-meteorological and isotopic studies along the EGIG } \\
\text { line, central Greenland., J. of Glaciol., 41, 139, 515-527, } 1995 .\end{array}$} \\
\hline 1990 & T09_1990 & 70.02 & -46.31 & -20.1 & 402 & 8.1 & 4.6 & \\
\hline 1990 & T13_1990 & 70.23 & -45.02 & -21.9 & 391 & 8.0 & 4.7 & \\
\hline 1990 & T17_1990 & 70.37 & -44.10 & -23.0 & 377 & 8.9 & 4.6 & \\
\hline 1990 & T21_1990 & 70.54 & -43.02 & -24.2 & 357 & 8.3 & 4.6 & \\
\hline 1990 & T27_1990 & 70.78 & -41.54 & -25.7 & 318 & 7.6 & 4.8 & \\
\hline 1990 & T31_1990 & 70.91 & -40.64 & -26.7 & 296 & 7.8 & 4.9 & \\
\hline 1990 & T41_1990 & 71.08 & -37.92 & -28.8 & 258 & 7.3 & 5.2 & \\
\hline 1990 & T43_1990 & 71.12 & -37.32 & -29.1 & 256 & 6.3 & 5.4 & \\
\hline 1990 & T43_1990 & 71.12 & -37.32 & -29.1 & 256 & 8.0 & 5.3 & \\
\hline 1990 & T47_1990 & 71.20 & -35.95 & -28.9 & 238 & 9.8 & 5.3 & \\
\hline 1990 & T53_1990 & 71.35 & -33.48 & -26.7 & 273 & 7.5 & 5.2 & \\
\hline 1990 & T61_1990 & 72.23 & -32.33 & -27.2 & 254 & 9.8 & 5.3 & \\
\hline 1990 & T99_1990 & 72.58 & -37.63 & -30.5 & 200 & 13.7 & 5.4 & \\
\hline 2011 & ACT11A2 & 66.18 & -39.02 & -12.7 & 1626 & 10.1 & 4.1 & \multirow{5}{*}{$\begin{array}{l}\text { Forster, R. R., Box, J. E., van den Broeke, M. R., Mièg } \\
\text { Burgess, E. W., Angelen, J. H., . . McConnell, J. R.: } \\
\text { Extensive liquid meltwater storage in firn within the } \\
\text { Greenland ice sheet., Nat. Geosci., 7, 95-19, } \\
\text { doi:10.1038/NGEO2043, 2014. }\end{array}$} \\
\hline 2011 & ACT11A & 66.18 & -39.08 & -13.2 & 1542 & 25.0 & 3.8 & \\
\hline 2011 & ACT11B & 66.22 & -39.57 & -14.3 & 1310 & 60.9 & 3.5 & \\
\hline 2011 & ACT11C & 66.29 & -40.75 & -17.0 & 983 & 61.4 & 4.0 & \\
\hline 2011 & ACT11D & 66.48 & -46.31 & -18.4 & 355 & 59.4 & 3.8 & \\
\hline 2008 & GGU163 & 69.73 & -48.19 & -16.7 & 445 & 10.5 & 2.8 & \multirow{8}{*}{$\begin{array}{l}\text { Harper, J., Humphrey, N., Pfeffer, W. T., Brown, J., and } \\
\text { Fettweis, X.: Greenland ice-sheet contribution to sea-level } \\
\text { rise buffered by meltwater storage in firn, Nature, } 491 \text {, } \\
240-243 \text {, doi:doi:10.1038/nature } 11566,2012 .\end{array}$} \\
\hline 2008 & GGU165 & 69.72 & -48.27 & -16.2 & 431 & 10.3 & 3.2 & \\
\hline 2008 & H1-1 & 69.74 & -48.24 & -16.7 & 445 & 11.1 & 3.2 & \\
\hline 2008 & H1-15 & 69.74 & -48.24 & -16.7 & 445 & 10.4 & 3.1 & \\
\hline 2008 & H1-30 & 69.74 & -48.24 & -16.7 & 445 & 10.3 & 3.0 & \\
\hline 2008 & $\mathrm{H} 2-1$ & 69.71 & -48.35 & -16.1 & 444 & 10.3 & 1.7 & \\
\hline 2008 & H3-1 & 69.69 & -48.50 & -15.4 & 413 & 10.3 & 2.5 & \\
\hline 2008 & H3.5-1 & 69.67 & -48.59 & -15.4 & 413 & 9.5 & 2.4 & \\
\hline
\end{tabular}




\begin{tabular}{|c|c|c|c|c|c|c|c|c|}
\hline 2008 & H4-1 & 69.66 & -48.69 & -15.2 & 406 & 10.0 & 1.5 & \\
\hline 2008 & H4-2 & 69.66 & -48.69 & -15.2 & 406 & 9.7 & 0.9 & \\
\hline 2008 & H5-1 & 69.64 & -48.82 & -14.9 & 407 & 8.2 & 0.5 & \\
\hline 2008 & T1-2 & 69.74 & -48.06 & -17.1 & 448 & 10.5 & 3.5 & \\
\hline 2007 & G1 & 69.88 & -47.01 & -19.0 & 420 & 9.7 & 4.5 & \\
\hline 2007 & G2 & 69.88 & -47.01 & -19.0 & 420 & 10.3 & 4.2 & \\
\hline 2007 & G3 & 69.88 & -47.01 & -19.0 & 420 & 10.4 & 4.7 & \\
\hline 2007 & G4 & 69.88 & -47.01 & -19.0 & 420 & 10.3 & 4.1 & \\
\hline 2007 & G5 & 69.88 & -47.01 & -19.0 & 420 & 10.1 & 4.5 & \\
\hline 2007 & G6 & 69.88 & -47.01 & -19.0 & 420 & 10.4 & 4.4 & \\
\hline 2007 & G7 & 69.88 & -47.01 & -19.0 & 420 & 10.2 & 4.7 & \\
\hline 2007 & G8 & 69.88 & -47.01 & -19.0 & 420 & 10.5 & 4.6 & \\
\hline 2007 & G9 & 69.88 & -47.01 & -19.0 & 420 & 10.4 & 4.6 & \\
\hline 2007 & SW-1 & 69.80 & -47.57 & -17.9 & 424 & 10.4 & 4.1 & \\
\hline 2007 & SW-2 & 69.80 & -47.57 & -17.9 & 424 & 10.2 & 4.0 & \\
\hline 2007 & SW-3 & 69.81 & -47.54 & -17.9 & 424 & 10.3 & 4.0 & \\
\hline 2007 & SW-4 & 69.79 & -47.61 & -17.9 & 426 & 10.2 & 4.1 & \\
\hline 2007 & T1-1 & 69.74 & -48.06 & -17.1 & 448 & 10.5 & 3.2 & \\
\hline 2007 & T2-1 & 69.76 & -47.88 & -17.3 & 443 & 10.5 & 4.1 & \\
\hline 2007 & T3-1 & 69.78 & -47.67 & -17.7 & 431 & 10.0 & 4.0 & \\
\hline 2007 & T3-2 & 69.78 & -47.67 & -17.7 & 431 & 10.4 & 3.7 & \\
\hline 2007 & T3-3 & 69.78 & -47.67 & -17.7 & 431 & 10.0 & 3.7 & \\
\hline 2007 & T4-1 & 69.82 & -47.45 & -18.2 & 425 & 10.6 & 4.2 & \\
\hline 2007 & T5-1 & 69.85 & -47.27 & -18.4 & 427 & 10.2 & 4.5 & \\
\hline 2011 & Hawley_2011 & 73.34 & -39.72 & -29.8 & 169 & 10.1 & 5.4 & \multirow{6}{*}{$\begin{array}{l}\text { Hawley, R. L., Courville, Z. R., Kehrl, L., Lutz, E., Osteberg, } \\
\text { E., Overly, T. B., and Wong, G.: Recent accumulation } \\
\text { variability in northwest Greenland from ground-penetrating } \\
\text { radar and shallow cores along the Greenland Inland Traverse, } \\
\text { J. Glaciol., 60, 220, 375-382, doi:10.3189/2014JoG13J141, } \\
2014 \text {. }\end{array}$} \\
\hline 2011 & Hawley_2011_2 & 74.02 & -40.62 & -30.4 & 156 & 10.4 & 5.5 & \\
\hline 2011 & Hawley_2011_3 & 74.42 & -39.29 & -30.8 & 116 & 10.0 & 4.9 & \\
\hline 2011 & Hawley_2011_10 & 76.50 & -43.73 & -31.2 & 128 & 10.5 & 5.0 & \\
\hline 2011 & Hawley_2011_11 & 76.50 & -44.84 & -30.7 & 154 & 10.2 & 5.3 & \\
\hline 2011 & Hawley_2011_16 & 77.26 & -58.53 & -25.7 & 400 & 7.0 & 4.8 & \\
\hline
\end{tabular}




$\begin{array}{llllllll}2011 & \text { Hawley_2011_19 } & 77.37 & -55.93 & -26.6 & 324 & 10.5 & 4.7 \\ 2011 & \text { Hawley_2011_22 } & 77.45 & -50.54 & -28.8 & 200 & 8.4 & 5.6\end{array}$

\begin{tabular}{|c|c|c|c|c|c|c|c|}
\hline 1993 U6 (2006) & 65.29 & -45.83 & -19.9 & 393 & 11.5 & 4.7 & $\begin{array}{l}\text { Summary. BPRC Technical Report No. 2012-01, Byrd Polar } \\
\text { Research Center, The Ohio State University, Columbus, Ohio, } \\
2012 .\end{array}$ \\
\hline
\end{tabular}

Jezek, K. C.: Surface Elevation and Velocity Changes on the Jezek, K. C.: Surface Elevation and Velocity Changes on the
South Central Greenland Ice Sheet: 1980-2011 - Data Research Center, The Ohio State University, Columbus, Ohio, 2012.

\begin{tabular}{|c|c|c|c|c|c|c|c|c|}
\hline 1989 & Site J 1989 & 66.87 & -46.26 & -18.4 & 354 & 206.6 & 4.2 & $\begin{array}{l}\text { Kameda, T., Narita, H., Shoji, H., Nishio, F., Fuji, Y., and } \\
\text { Watanabe, O.: Melt features in ice cores from Site J, southern } \\
\text { Greenland: some implication for summer climate since AD } \\
\text { 1550, Ann. Glaciol., 21, 51-58, } 1995 \text {. }\end{array}$ \\
\hline 2013 & FA13A & 66.18 & -39.04 & -12.7 & 1626 & 50.4 & 3.8 & \multirow{2}{*}{$\begin{array}{l}\text { Koenig, L. S., Miège, C., Forster, R. R., and Brucker, L.: } \\
\text { Initial in situ measurements of perennial meltwater storage in } \\
\text { the Greenland firn aquifer, Geophys. Res. Lett., 41, 81-85, } \\
\text { doi:10.1002/2013GL058083, } 2014 \text {. }\end{array}$} \\
\hline 2013 & FA13B & 66.18 & -39.04 & -12.7 & 1626 & 32.1 & 3.9 & \\
\hline 1963 & Camp Century & 77.18 & -61.17 & -24.1 & 385 & 222.6 & 4.8 & $\begin{array}{l}\text { Kovacs, A., Weeks, W. F., and Michitti, F.: Variation of Some } \\
\text { Mechanical Properties of Polar Snow, Camp Century, } \\
\text { Greenland, CRREL Res. Rpt. 276, } 1969 .\end{array}$ \\
\hline 1965 & Site 2 & 76.98 & -56.07 & -24.6 & 397 & 282.5 & 4.7 & $\begin{array}{l}\text { Langway, C. C.: Stratigraphic analysis of a deep ice core from } \\
\text { Greenland, CRREL Res. Rpt. 77, } 1967 .\end{array}$ \\
\hline 2007 & Albert_2007 & 72.58 & -38.50 & -30.2 & 204 & 86.9 & 5.0 & $\begin{array}{l}\text { Lomonaco, R., Albert, M., and Baker, I.: Microstructural } \\
\text { evolution of fine-grained layers through the firn column at } \\
\text { Summit, Greenland, J. Glaciol., 57, 204, } 2011 .\end{array}$ \\
\hline 2015 & core_2_2015 & 66.99 & -44.39 & -21.0 & 380 & 15.6 & 4.4 & \multirow{9}{*}{$\begin{array}{l}\text { Machguth, H., MacFerrin, M., As, D. v., Box, J., } \\
\text { Charalampidis, C., Colgan, W., . . Mosley-Thompson, E.: } \\
\text { Greenland meltwater storage in firn limited by near-surface } \\
\text { ice formation, Nature Clim. Change, 6, 390-395, } \\
\text { doi:10.1038/NCLIMATE2899, 2016. }\end{array}$} \\
\hline 2015 & core_3_2015 & 66.48 & -42.50 & -20.7 & 690 & 16.6 & 4.3 & \\
\hline 2015 & core_5_2015 & 66.48 & -42.50 & -20.7 & 690 & 8.3 & 4.4 & \\
\hline 2015 & core_6_2015 & 66.48 & -42.50 & -20.7 & 690 & 16.3 & 4.4 & \\
\hline 2015 & core_7_2015 & 66.00 & -44.50 & -21.0 & 519 & 16.4 & 4.3 & \\
\hline 2015 & core_9_2015 & 66.00 & -44.50 & -21.0 & 519 & 8.3 & 4.4 & \\
\hline 2015 & core_10_2015 & 66.00 & -44.50 & -21.0 & 519 & 16.5 & 4.3 & \\
\hline 2015 & core_11_2015 & 66.48 & -46.29 & -18.4 & 355 & 19.3 & 3.3 & \\
\hline 2015 & core_14_2015 & 69.88 & -47.03 & -19.0 & 420 & 17.3 & 4.2 & \\
\hline
\end{tabular}




\begin{tabular}{|c|c|c|c|c|c|c|c|c|}
\hline 2015 & core_16_2015 & 69.88 & -47.03 & -19.0 & 420 & 9.4 & 4.0 & \\
\hline 2015 & core_18_2015 & 75.63 & -35.98 & -30.6 & 94 & 15.3 & 5.4 & \\
\hline 2015 & core_20_2015 & 75.63 & -35.98 & -30.6 & 94 & 8.0 & 5.6 & \\
\hline 2015 & core_22_2015 & 72.58 & -38.47 & -30.3 & 203 & 15.8 & 5.4 & \\
\hline 2015 & core_24_2015 & 72.58 & -38.47 & -30.3 & 203 & 7.7 & 5.5 & \\
\hline 2015 & core_25_2015 & 72.58 & -38.47 & -30.3 & 203 & 15.9 & 5.4 & \\
\hline 2013 & core_1_2013 & 67.00 & -47.02 & -16.7 & 348 & 19.1 & 1.2 & \\
\hline 2013 & core_2_2013 & 67.00 & -47.02 & -16.7 & 348 & 15.9 & 1.0 & \\
\hline 2013 & core_3_2013 & 66.98 & -46.63 & -17.8 & 362 & 16.0 & 2.4 & \\
\hline 2013 & core_4_2013 & 66.98 & -46.12 & -18.5 & 358 & 16.3 & 3.0 & \\
\hline 2013 & core_5_2013 & 66.48 & -46.28 & -18.4 & 355 & 16.6 & 3.3 & \\
\hline 2013 & core_6_2013 & 66.47 & -46.28 & -18.4 & 355 & 16.5 & 3.0 & \\
\hline 2013 & core_7_2013 & 66.98 & -45.75 & -19.2 & 359 & 16.4 & 3.6 & \\
\hline 2013 & core_8_2013 & 66.98 & -45.04 & -20.3 & 363 & 16.3 & 3.8 & \\
\hline 2013 & core_9_2013 & 66.99 & -44.39 & -21.0 & 380 & 17.0 & 4.3 & \\
\hline 2012 & core_1_2012 & 67.00 & -47.02 & -16.7 & 348 & 10.7 & 1.8 & \\
\hline 2012 & core_2_2012 & 67.00 & -47.02 & -16.7 & 348 & 10.5 & 1.9 & \\
\hline 2012 & core_3_2012 & 67.00 & -47.02 & -16.7 & 348 & 10.2 & 1.6 & \\
\hline 1984 & Mayewski_1984 & 65.10 & -44.87 & -20.8 & 472 & 104.1 & 4.7 & $\begin{array}{l}\text { Mayewski, P., and Whitlow, S.: 2016. Snow Pit and Ice Core } \\
\text { Data from Southern Greenland, 1984, NSF Arctic Data } \\
\text { Center. doi:10.5065/D6S180MH, 2016. }\end{array}$ \\
\hline 1990 & Mayewski_1990 & 72.58 & -38.46 & -30.3 & 203 & 6.1 & 5.3 & $\begin{array}{l}\text { Mayewski, P., and Whitlow S.: Snow Pit Data from Greenland } \\
\text { Summit, 1989 to 1993. NSF Arctic Data Center. } \\
\text { doi:10.5065/D6NP22KX, 2016. }\end{array}$ \\
\hline 2010 & ACT10A & 65.70 & -41.48 & -15.0 & 1281 & 46.6 & 4.1 & Miège, C., Forster R.C., B. J., Burgess, E., McConnell, J., \\
\hline 2010 & ACT10B & 65.78 & -41.87 & -16.5 & 1101 & 50.8 & 4.7 & $\begin{array}{l}\text { Pasteris, D., and Spikes, V. B.: Southeast Greenland high } \\
\text { accumulation rates derived from firn cores and ground- }\end{array}$ \\
\hline 2010 & ACT10C & 66.00 & -42.78 & -19.5 & 838 & 49.1 & 4.4 & $\begin{array}{l}\text { penetrating radar, Ann. Glaciol., 54, 63, 322-332, } \\
\text { doi:10.3189/2013AoG63A358, } 2013 .\end{array}$ \\
\hline 2011 & T21_2011 & 70.54 & -43.02 & -24.2 & 357 & 9.5 & 4.5 & \multirow{4}{*}{$\begin{array}{l}\text { Morris, E. M., and Wingham, D. J.: Densification of polar } \\
\text { snow: Meaurements, modeling and implication for altimetry, } \\
\text { J. Geophys. Res.-Earth, doi:10.1002/2013JF002898, } 2014 \text {. }\end{array}$} \\
\hline 2011 & T21c_2011 & 70.54 & -43.02 & -24.2 & 357 & 8.2 & 4.5 & \\
\hline 2011 & T23_2011 & 70.63 & -42.58 & -24.6 & 344 & 8.6 & 4.8 & \\
\hline 2011 & T27_2011 & 70.78 & -41.54 & -25.7 & 318 & 8.4 & 4.8 & \\
\hline
\end{tabular}




\begin{tabular}{ll}
2011 & T31_2011 \\
2011 & T39_2011 \\
2011 & T41_2011 \\
2011 & T41b_2011 \\
2011 & T41c_2011 \\
2011 & T41d_2011 \\
2010 & T21_2010 \\
2010 & T21b_2010 \\
2010 & T23_2010 \\
2010 & T27_2010 \\
2010 & T31_2010 \\
2010 & T35_2010 \\
2010 & T39_2010 \\
2010 & T41_2010 \\
2010 & T41b_2010 \\
2010 & T41c_2010 \\
2010 & T41e_2010 \\
2006 & T41_2006 \\
2006 & T41b_2006 \\
2006 & T41c_2006 \\
2006 & T41d_2006 \\
2006 & T12_2006 \\
2006 & T15_2006 \\
2006 & T19_2006 \\
2006 & T31_2006 \\
2006 & T35_2006 \\
2006 & T41e_2006 \\
2006 & T12b_2006 \\
2006 & T15b_2006 \\
2006 & T19b_2006 \\
2006 & T21_2006 \\
& \\
\hline
\end{tabular}

$\begin{array}{lccccc}70.91 & -40.64 & -26.7 & 296 & 9.8 & 4.8 \\ 71.04 & -38.46 & -28.4 & 263 & 6.8 & 4.8 \\ 71.08 & -37.92 & -28.8 & 258 & 6.8 & 5.1 \\ 71.08 & -37.92 & -28.8 & 258 & 7.3 & 5.0 \\ 71.08 & -37.92 & -28.8 & 258 & 7.0 & 5.1 \\ 71.08 & -37.92 & -28.8 & 258 & 6.2 & 5.2 \\ 70.54 & -43.02 & -24.2 & 357 & 10.2 & 4.7 \\ 70.54 & -43.02 & -24.2 & 357 & 10.0 & 4.7 \\ 70.63 & -42.58 & -24.6 & 344 & 6.9 & 4.9 \\ 70.78 & -41.54 & -25.7 & 318 & 8.9 & 4.9 \\ 70.91 & -40.64 & -26.7 & 296 & 9.9 & 4.8 \\ 70.98 & -39.55 & -27.6 & 275 & 8.0 & 5.1 \\ 71.04 & -38.46 & -28.4 & 263 & 8.8 & 5.0 \\ 71.08 & -37.92 & -28.8 & 258 & 9.3 & 5.0 \\ 71.08 & -37.92 & -28.8 & 258 & 7.1 & 5.3 \\ 71.08 & -37.92 & -28.8 & 258 & 8.7 & 5.2 \\ 71.08 & -37.92 & -28.8 & 258 & 5.7 & 5.4 \\ 71.08 & -37.92 & -28.8 & 258 & 9.4 & 5.0 \\ 71.08 & -37.92 & -28.8 & 258 & 10.3 & 5.1 \\ 71.08 & -37.92 & -28.8 & 258 & 8.9 & 5.1 \\ 71.08 & -37.92 & -28.8 & 258 & 8.9 & 5.2 \\ 70.18 & -45.34 & -21.5 & 398 & 9.1 & 4.6 \\ 70.30 & -44.57 & -22.4 & 388 & 11.4 & 4.6 \\ 70.47 & -43.56 & -23.6 & 369 & 12.0 & 4.5 \\ 70.91 & -40.64 & -26.7 & 296 & 9.1 & 4.9 \\ 70.98 & -39.55 & -27.6 & 275 & 9.4 & 4.9 \\ 71.08 & -37.92 & -28.8 & 258 & 10.5 & 5.0 \\ 70.18 & -45.34 & -21.5 & 398 & 7.2 & 4.7 \\ 70.30 & -44.57 & -22.4 & 388 & 11.7 & 4.6 \\ 70.47 & -43.56 & -23.6 & 369 & 12.1 & 4.5 \\ 70.54 & -43.02 & -24.2 & 357 & 8.6 & 4.7\end{array}$




\begin{tabular}{llllllll}
2006 & T23_2006 & 70.63 & -42.58 & -24.6 & 344 & 6.7 & 4.8 \\
2006 & T27_2006 & 70.78 & -41.54 & -25.7 & 318 & 8.2 & 4.7 \\
2006 & T31b_2006 & 70.91 & -40.64 & -26.7 & 296 & 8.5 & 4.9 \\
2006 & T35b_2006 & 70.98 & -39.55 & -27.6 & 275 & 9.5 & 4.8 \\
2006 & T39_2006 & 71.04 & -38.46 & -28.4 & 263 & 6.4 & 5.0 \\
2006 & T41f_2006 & 71.08 & -37.92 & -28.8 & 258 & 10.3 & 5.0 \\
2006 & T05_2006 & 69.85 & -47.25 & -18.7 & 425 & 11.0 & 4.1 \\
2006 & T09_2006 & 70.02 & -46.31 & -20.1 & 402 & 10.1 & 4.5 \\
2006 & T21b_2006 & 70.54 & -43.02 & -24.2 & 357 & 9.4 & 4.6 \\
2006 & T41g_2006 & 71.08 & -37.92 & -28.8 & 258 & 9.5 & 5.0 \\
2006 & T41h_2006 & 71.08 & -37.92 & -28.8 & 258 & 10.4 & 5.0 \\
2006 & T41i_2006 & 71.08 & -37.92 & -28.8 & 258 & 9.0 & 5.1 \\
2006 & T41j_2006 & 71.08 & -37.92 & -28.8 & 258 & 8.8 & 5.2 \\
2004 & T41_Spring_2004 & 71.08 & -37.92 & -28.8 & 258 & 11.4 & 5.0 \\
2004 & T41b_Spring_2004 & 71.08 & -37.92 & -28.8 & 258 & 11.1 & 5.1 \\
2004 & T41c_Spring_2004 & 71.08 & -37.92 & -28.8 & 258 & 10.6 & 5.1 \\
2004 & T41d_Spring_2004 & 71.08 & -37.92 & -28.8 & 258 & 11.2 & 5.2 \\
2004 & T41_Autumn_2004 & 71.08 & -37.92 & -28.8 & 258 & 10.2 & 5.0 \\
2004 & T41b_Autumn_2004 & 71.08 & -37.92 & -28.8 & 258 & 10.0 & 5.0 \\
2004 & T41c_Autumn_2004 & 71.08 & -37.92 & -28.8 & 258 & 10.7 & 5.1 \\
2004 & T41d_Autumn_2004 & 71.08 & -37.92 & -28.8 & 258 & 11.8 & 4.9 \\
2004 & T12_Spring_2004 & 70.18 & -45.34 & -21.5 & 398 & 12.7 & 4.5 \\
2004 & T15_Spring_2004 & 70.30 & -44.57 & -22.4 & 388 & 12.0 & 4.6 \\
2004 & T21_Spring_2004 & 70.54 & -43.02 & -24.2 & 357 & 13.8 & 4.6 \\
2004 & T23_Spring_2004 & 70.63 & -42.58 & -24.6 & 344 & 12.6 & 4.6 \\
2004 & T27_Spring_2004 & 70.78 & -41.54 & -25.7 & 318 & 12.0 & 4.8 \\
2004 & T31_Spring_2004 & 70.91 & -40.64 & -26.7 & 296 & 12.3 & 4.8 \\
2004 & T35_Spring_2004 & 70.98 & -39.55 & -27.6 & 275 & 11.9 & 4.9 \\
2004 & T39_Spring_2004 & 71.04 & -38.46 & -28.4 & 263 & 11.2 & 4.9 \\
2004 & T41e_Spring_2004 & 71.08 & -37.92 & -28.8 & 258 & 12.4 & 5.0 \\
2004 & T21b_Spring_2004 & 70.54 & -43.02 & -24.2 & 357 & 12.1 & 4.7 \\
\hline & & & & & & &
\end{tabular}




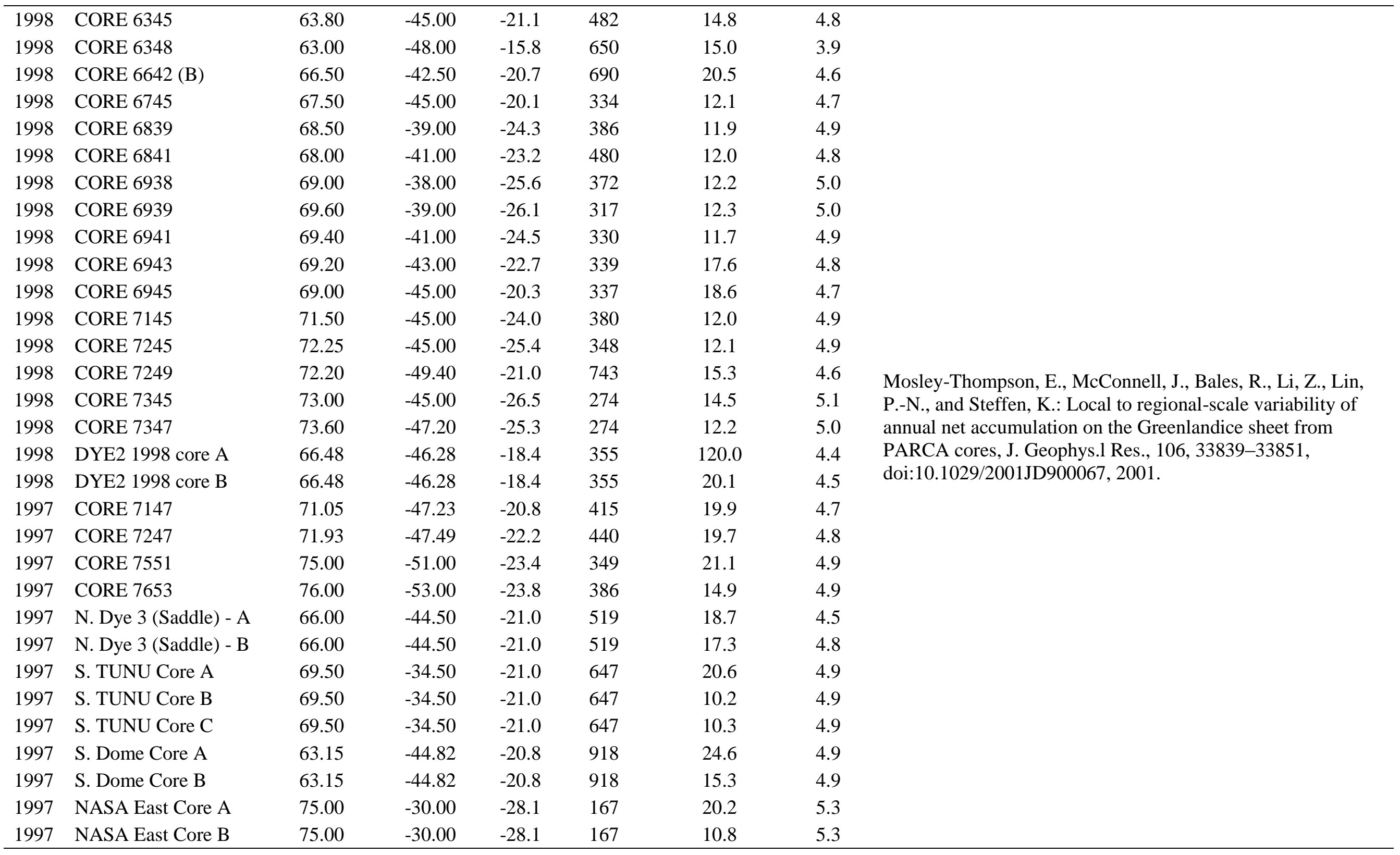




\begin{tabular}{|c|c|c|c|c|c|c|c|c|}
\hline 2007 & CP_2007 & 70.00 & -47.00 & -19.2 & 411 & 152.1 & 4.7 & $\begin{array}{l}\text { Porter, S., and Mosley-Thompson, E.: Exploring seasonal } \\
\text { accumulation bias in a west central Greenland ice core with } \\
\text { observed and reanalyzed data, J. Glaciol., 60, 224, 1065-1074, } \\
\text { doi:10.3189/2014JoG13J233, 2014. }\end{array}$ \\
\hline 1963 & DYE-2 1963 & 66.47 & -46.28 & -18.4 & 355 & 31.8 & 4.1 & $\begin{array}{l}\text { Reed, S.: Performance Study of the Dewline Ice Cap Stations, } \\
\text { 1963, CRREL Special Report 72, } 1966 .\end{array}$ \\
\hline 1967 & Carrefour_1967 & 69.82 & -47.43 & -18.2 & 425 & 19.8 & 4.2 & \multirow{7}{*}{$\begin{array}{l}\text { Renaud, A.: Etude physiques et chimiques sur la glace de } \\
\text { l'indlandsis du Groenland, Medd. Groenland, 2, 177, 100-107, } \\
1959 .\end{array}$} \\
\hline 1959 & Camp_VI_1959 & 69.70 & -48.27 & -16.1 & 444 & 39.5 & 4.1 & \\
\hline 1959 & Milcent_1959 & 70.30 & -44.57 & -22.4 & 388 & 20.2 & 4.7 & \\
\hline 1959 & Centrale_1959 & 70.91 & -40.64 & -26.7 & 296 & 30.9 & 4.7 & \\
\hline 1959 & Crete_1959 & 71.12 & -37.32 & -29.1 & 256 & 15.8 & 5.0 & \\
\hline 1959 & Jarl-Joset_1959 & 71.35 & -33.48 & -26.7 & 273 & 27.0 & 4.9 & \\
\hline 1959 & Depot 420_1959 & 72.23 & -32.33 & -27.2 & 254 & 13.8 & 4.9 & \\
\hline 1990 & GRIP & 72.57 & -37.62 & -30.5 & 202 & 82.3 & 5.3 & \multirow{8}{*}{$\begin{array}{l}\text { Spencer, M. K., Aller, R. B., and Creyts, T. T.: Preliminary } \\
\text { firn-densification model with 38-site dataset, J. Glaciol., 47, } \\
\text { 159, 671-676, } 2001 \text {. }\end{array}$} \\
\hline 1984 & Dye3-11B-1984 & 65.18 & -43.83 & -20.2 & 620 & 24.8 & 4.9 & \\
\hline 1984 & Dye3-15B-1984 & 65.18 & -43.83 & -20.2 & 620 & 24.8 & 4.9 & \\
\hline 1984 & Dye3-16C-1984 & 65.18 & -43.83 & -20.2 & 620 & 24.8 & 4.9 & \\
\hline 1983 & Dye3-4B-1983 & 65.18 & -43.83 & -20.2 & 620 & 173.6 & 4.8 & \\
\hline 1984 & Dye3-5B-1984 & 65.18 & -43.83 & -20.2 & 620 & 24.8 & 4.8 & \\
\hline 1984 & Dye3-9B-1984 & 65.18 & -43.83 & -20.2 & 620 & 24.8 & 4.8 & \\
\hline 1983 & Dye3-station1-1983 & 65.18 & -43.83 & -20.2 & 620 & 27.1 & 4.8 & \\
\hline 2007 & NEEM07S3 & 77.50 & -51.00 & -28.7 & 205 & 80.0 & 5.2 & $\begin{array}{l}\text { Steen-Larsen, H. C., Masson-Delmotte, V., Sjolte, J., Johnsen, } \\
\text { S. J., Vinther, B. M., Bréon, F.-M., . . ...: Understanding the } \\
\text { climatic signal in the water stable isotope records from the } \\
\text { NEEM cores, J. Geophys. Res., 116, D06108, } \\
\text { doi:10.1029/2010JD014311, 2011. }\end{array}$ \\
\hline 2012 & NEGIS & 75.62 & -35.96 & -30.6 & 94 & 66.3 & 5.8 & $\begin{array}{l}\text { Vallelonga, P., Christianson, K., Alley, R. B., } \\
\text { Anandakrishnan, S., Christian, J. E., Dahl-Jensen, D., . . } \\
\text { Popp, T.: Initial results from geophysical surveys and shallow } \\
\text { coring of the Northeast Greenland Ice Stream (NEGIS), } \\
\text { Cryosphere, 8, 1275-1287, doi:10.5194/tc-8-1275-2014, } 2014 .\end{array}$ \\
\hline 1987 & Summit & 72.29 & -37.92 & -30.4 & 215 & 16.3 & 5.1 & van der Veen, C. J., Mosley-Thompson, E., Jezek, K. C., \\
\hline 1981 & L1 (1001) & 65.39 & -47.67 & -16.5 & 419 & 20.0 & 3.2 & Whillans, I. M., and Bolzan, J. F.: Accumulation rates in \\
\hline
\end{tabular}




\begin{tabular}{|c|c|c|c|c|c|c|c|c|}
\hline 1981 & L2 (12.18) & 65.39 & -47.19 & -17.6 & 398 & 20.1 & 3.8 & South and Central Greenland, Polar Geography, 25, 2, 79-162, \\
\hline 1981 & L5 (15.12) & 65.40 & -47.85 & -16.2 & 418 & 20.7 & 2.4 & \\
\hline 1981 & U3 (2003) & 64.93 & -45.60 & -20.3 & 403 & 15.1 & 4.4 & \\
\hline 1981 & U4 (2004) & 64.98 & -46.03 & -19.8 & 395 & 16.0 & 4.1 & \\
\hline 1981 & U6 (2006) & 65.29 & -45.83 & -19.9 & 393 & 15.0 & 4.2 & \\
\hline 1981 & U7 (2007) & 65.25 & -45.41 & -20.4 & 408 & 15.1 & 4.5 & \\
\hline 1981 & 1D (3001) & 65.25 & -43.49 & -19.3 & 750 & 8.7 & 3.7 & \\
\hline 1981 & 8D (3008) & 64.85 & -44.65 & -20.9 & 504 & 18.3 & 4.3 & \\
\hline 1995 & B26_NGT37_1995 & 77.15 & -49.13 & -29.4 & 198 & 118.7 & 5.5 & \multirow{13}{*}{$\begin{array}{l}\text { Wilhelms, F.: Measuring the Conductivity and Density of Ice } \\
\text { Cores, Ber. Polarforsch., 191, } 1996 .\end{array}$} \\
\hline 1995 & B27_NGT39_1995 & 76.39 & -46.29 & -29.9 & 192 & 172.4 & 5.3 & \\
\hline 1995 & B28_NGT39_1995 & 76.39 & -46.29 & -29.9 & 192 & 70.1 & 5.4 & \\
\hline 1995 & B29_NGT42_1995 & 76.00 & -43.29 & -30.9 & 144 & 108.2 & 5.8 & \\
\hline 1995 & B30_NGT45_1995 & 75.00 & -42.00 & -30.8 & 150 & 163.9 & 5.4 & \\
\hline 1994 & B19_NGT19_1994 & 78.00 & -36.23 & -29.5 & 83 & 149.4 & 5.6 & \\
\hline 1994 & B20_NGT23_1994 & 78.50 & -36.30 & -29.7 & 86 & 149.7 & 5.5 & \\
\hline 1994 & B21_NGT27_1994 & 80.00 & -41.08 & -29.6 & 121 & 100.4 & 5.6 & \\
\hline 1994 & B22_NGT30_1994 & 79.20 & -45.54 & -29.6 & 134 & 119.6 & 5.6 & \\
\hline 1994 & B23_NGT33_1994 & 78.00 & -44.00 & -31.3 & 115 & 150.2 & 5.7 & \\
\hline 1993 & B16_NGT03_1993 & 73.56 & -37.37 & -30.3 & 135 & 101.6 & 5.6 & \\
\hline 1993 & B17_NGT06_1993 & 75.15 & -37.37 & -31.1 & 92 & 100.4 & 5.6 & \\
\hline 1993 & B18_NGT14_1993 & 76.37 & -36.24 & -30.0 & 88 & 149.0 & 5.5 & \\
\hline 2017 & core_1_2017 & 67.00 & -47.02 & -16.7 & 348 & 23.3 & 0.9 & \multirow{10}{*}{ This study } \\
\hline 2017 & core_2_2017 & 66.99 & -44.39 & -21.0 & 380 & 22.2 & 4.2 & \\
\hline 2017 & core_3_2017 & 66.48 & -42.50 & -20.7 & 690 & 22.5 & 4.4 & \\
\hline 2017 & core_4_2017 & 66.00 & -44.50 & -21.0 & 519 & 22.7 & 4.6 & \\
\hline 2017 & core_5_2017 & 66.48 & -46.29 & -18.4 & 355 & 23.0 & 3.3 & \\
\hline 2017 & core_6_2017 & 66.48 & -46.29 & -18.4 & 355 & 7.0 & 3.2 & \\
\hline 2017 & core_7_2017 & 66.48 & -46.29 & -18.4 & 355 & 5.6 & 3.3 & \\
\hline 2017 & core_8_2017 & 66.63 & -46.89 & -17.2 & 330 & 15.2 & 2.0 & \\
\hline 2017 & core_9_2017 & 69.88 & -47.03 & -19.0 & 420 & 22.3 & 3.9 & \\
\hline 2017 & core_10_2017 & 75.63 & -35.98 & -30.6 & 94 & 20.1 & 5.4 & \\
\hline
\end{tabular}




$\begin{array}{llllllll}2017 & \text { core_11_2017 } & 72.58 & -38.47 & -30.3 & 203 & 22.2 & 5.2 \\ 2016 & \text { core_1_2016 } & 67.00 & -47.03 & -16.7 & 348 & 8.0 & 1.1 \\ 2016 & \text { core_2_2016 } & 67.00 & -47.03 & -16.7 & 348 & 16.5 & 1.6 \\ 2016 & \text { core_3_2016 } & 66.99 & -44.39 & -21.0 & 380 & 18.0 & 4.7 \\ 2016 & \text { core_4_2016 } & 66.48 & -42.50 & -20.7 & 690 & 18.4 & 4.7 \\ 2016 & \text { core_5_2016 } & 66.00 & -44.50 & -21.0 & 519 & 18.5 & 4.5 \\ 2016 & \text { core_6_2016 } & 72.58 & -38.47 & -30.3 & 203 & 16.3 & 5.5 \\ 2016 & \text { core_7_2016 } & 75.63 & -35.98 & -30.6 & 94 & 15.9 & 5.4 \\ 2016 & \text { core_8_2016 } & 69.88 & -47.03 & -19.0 & 420 & 18.1 & 3.9 \\ 2016 & \text { core_10_2016 } & 66.48 & -46.29 & -18.4 & 355 & 17.4 & 3.3\end{array}$

